\title{
Comparação de Técnicas FTIR de Transmissão, Reflexão e Fotoacústica na Análise de Poliamida-6, Reciclada e Irradiada
}

\author{
Maria C. Evora; Odair L. Gonçalez \\ Instituto de Estudos Avançados, CTA
}

Rita C.L.Dutra, Milton F. Diniz

Instituto de Aeronáutica e Espaço, CTA

\author{
Helio Wiebeck \\ Escola Politécnica, USP
}

\section{Leonardo G. de Andrade e Silva IPEN-CNEN}

Resumo: Um estudo comparativo envolvendo técnicas de análise por espectroscopia no infravermelho com transformada de Fourier (FTIR) de transmissão, reflexão e fotoacústica é apresentado com análise de poliamida-6. O potencial e as limitações destes métodos são investigados para analisar as alterações espectrométricas que ocorrem no interior e na superfície da poliamida-6, reciclada, e irradiada na presença de oxigênio, com feixe de elétrons de $1,5 \mathrm{MeV}$, na dose de $500 \mathrm{kGy}$. As técnicas mostram-se sensíveis para detectar pequenas mudanças de estruturas que ocorrem na poliamida- 6 reciclada e irradiada. Os espectros sugerem formação de grupos $-\mathrm{OH}, \mathrm{HOC}=\mathrm{O}-$, e $-\mathrm{C}=\mathrm{O}$, e mudanças estruturais relacionadas com grupos $-\mathrm{NH}$ e $-\mathrm{CN}-\mathrm{C}=\mathrm{O}$. Basicamente, as mesmas alterações espectrométricas são observadas com o uso das técnicas, sendo mais evidenciadas no interior das amostras pela espectroscopia-FTIR de transmissão, e na sua superfície, pela microscopia-FTIR.

Palavras-chave: Poliamida-6 reciclada, poliamida-6 irradiada, espectroscopia no infravermelho

\section{A Comparison of Transmission, Reflection and Photoacoustic FTIR Techniques in the Analysis of Recycled and Irradiated Polyamide-6}

\begin{abstract}
A comparative study involving transmission, reflection and photoacoustic FTIR techniques is presented with analysis of polyamide- 6 . The potential and limitations of these methods are investigated by analyzing structural variations that take place at the surface in the bulk in recycled and irradiated polyamide6 with a $1.5 \mathrm{MeV}$ electron beam with a $500 \mathrm{kGY}$ dose, in the presence of $\mathrm{O}_{2}$. FTIR techniques appear to be sensitive in detecting small structural changes that occur in recycled and irradiated polyamide- 6 . The analysis of samples indicated the formation of $-\mathrm{OH}, \mathrm{HOC}=\mathrm{O}-,-\mathrm{C}=\mathrm{O}$ groups. Also, small structural changes were detected which are characterisitic of $\mathrm{NH}$ and $-\mathrm{CN}-\mathrm{C}=\mathrm{O}$ groups. Transmission techniques show better the structural changes in the bulk, and microscopy-FTIR appears to be more sensitive in detecting what occurs at the sample surface.
\end{abstract}

Keywords: Recycled polyamide-6, irradiated polyamide-6, infrared spectroscopy

Autor para correspondência: Leonardo G. de Andrade e Silva, IPEN/CNEN, Travessa R, no 40, Cidade Universitária, CEP: 05508-900, São Paulo, SP. E-mail: Igasilva@net.ipen.br 


\section{Introdução}

A radiação ionizante, ao interagir com polímeros, transfere energia aos átomos da cadeia polimérica, provocando modificações permanentes na sua estrutura físico-química. Tais modificações podem resultar na reticulação ou na cisão das cadeias poliméricas, que são processos simultâneos e concorrentes, e cuja preponderância de um ou outro depende principalmente da dose de radiação com que foi tratado o material.

$\mathrm{Na}$ irradiação com feixes de elétrons, os elétrons acelerados em baixas energias $(\mathrm{E}<8 \mathrm{MeV})$, depois de penetrarem na matéria, interagem eletrostaticamente com elétrons e núcleos atômicos, sofrendo atração e repulsão. Muitos elétrons são removidos do feixe na interação com elétrons orbitais pela excitação e ionização dos átomos do meio ${ }^{[1]}$.

As moléculas poliméricas quando são irradiadas, liberam elétrons de alta energia e provocam ionização da molécula ${ }^{[2]}$.

$$
\mathrm{M} \stackrel{\text { radiação }}{\longrightarrow} \mathrm{MM}^{+}+\mathrm{e}^{-}
$$

Este elétron primário causa ionização de outras moléculas produzindo mais elétrons.

$$
\mathrm{e}^{-}+\mathrm{MM} \longrightarrow \mathrm{MM}^{+}+2 \mathrm{e}^{-}
$$

Os íons carregados positivamente, desaparecem rapidamente ao combinarem com elétrons. Isto produz um estado eletrônico altamente excitado.

$$
\mathrm{MM}^{+}+\mathrm{e}^{-} \longrightarrow \mathrm{MM}^{*}
$$

Uma parte das interações da radiação com o material polimérico pode também resultar diretamente em transferência de energia, que não é suficiente para causar ionização, mas resulta diretamente em um estado eletrônicamente excitado.

$$
\mathrm{MM} \stackrel{\text { radiação }}{\longrightarrow} \mathrm{MM}^{*}
$$

Estas moléculas que se encontram no estado excitado decaem para o estado fundamental emitindo fosforescência e fluorescência ou por meio de reações químicas, por quebra heterolítica da ligação produzindo íons, ou por quebra homolítica de ligações, favorecendo a formação de radicais, onde ocorrerá o processo de reticulação.

$$
\mathrm{MM}^{*} \longrightarrow \mathrm{M} \mathrm{M}
$$

O processo de reticulação introduz ligações cruzadas, ou seja, ligações entre moléculas lineares produzindo polímeros tridimensionais com alta massa molar. Com o aumento da reticulação, a estrutura se torna mais rígida ${ }^{[1]}$.

O grau de reticulação é proporcional a dose absorvida, sendo independente da intensidade da radiação ${ }^{[3]}$.

A poliamida-6 é um polímero semicristalino com alto grau de cristalinidade. Devido à sua alta performance, a poliamida- 6 , também conhecida como nylon-6, é um dos mais importantes termoplásticos de engenharia. Tem ampla aplicação, substituindo o metal em componentes e conexões que necessitam de excelentes propriedades mecânicas, químicas e físicas.

A reciclagem da poliamida- 6 já é bastante difundida, mas a aplicação deste polímero reciclado é limitada. Sucessivas reciclagens promovem alterações nas propriedades de diferentes materiais poliméricos. Sabe-se que, em geral, ocorrem quebras de estruturas, diminuição de massa molar, aumento do índice de fluidez e diminuição da resistência mecânica. Tais alterações dependem muito do tipo de resina plástica reciclada, das operações envolvidas, da duração do processo, bem como da temperatura e da tensão de cisalhamento aplicadas ${ }^{[4]}$. O ideal é que a poliamida6 reciclada seja utilizada como matéria prima de novos produtos com características adequadas de qualidade, tornando-os competitivos frente à matéria prima proveniente da indústria petroquímica.

Singh e Chaudhuri ${ }^{[5]}$ estudaram os efeitos da radiação $\gamma$ sobre a poliamida-6, utilizando o método de intumescimento. Concluíram que acima de $36 \mathrm{kGy}$ ocorrem tanto o processo de degradação, quanto a reticulação. Gupta e Pandey ${ }^{[6]}$ observaram que a condutividade elétrica aumenta quando a poliamida6 é irradiada com radiação $\gamma$.

Até então, os trabalhos existentes sobre os efeitos da radiação $g$ sobre a poliamida-6, tratam do material não reciclado. Em trabalho anterior, Evora et a ${ }^{[7]}$ submeteram as amostras de poliamida- 6 recicladas à irradiação com feixes de elétrons nas doses de 50, 100, 200, 300 e $500 \mathrm{kGy}$, a uma taxa de $22,61 \mathrm{kGy} / \mathrm{s}$. Foram feitos ensaios de termogravimetria (TG) e calorimetria exploratória diferencial (DSC) que permitiram o acompanhamento do processo de reticulação em função da dose de radiação.

Outras técnicas, tal como a espectroscopia FTIR 
pode ser usada para identificação e caracterização de compostos orgânicos, inorgânicos e poliméricos. Basicamente o que se mede nesta análise, é a fração da energia transmitida ou absorvida em relação à incidente em determinado comprimento de onda ou número de onda ${ }^{[8]}$. Diversos estudos foram publicados, devido à habilidade da técnica para detectar mudanças estruturais decorrentes de diferentes tratamentos, entre eles inclui-se os referentes às poliamidas $^{[9-12]}$.

A aplicação de técnicas de preparação de amostras (transmissão e reflexão) para análise por espectroscopia no infravermelho médio (MIR) (4000 a $666 \mathrm{~cm}^{-1}$ ) com transformada de Fourier (FTIR), é uma boa prática para avaliação mais completa dos materiais ${ }^{[9,13,14]}$, ou seja, análise do interior e superfície. Técnicas de detecção fotoacústica (PAS) podem ser utilizadas quando as amostras são de difícil preparo e outras técnicas falham ou fornecem um pequeno número de informações ${ }^{[15]}$.

A avaliação de bandas na região infravermelho próximo (NIR) ${ }^{[16]}(0,7$ a $3 \mu \mathrm{m}$ ou 700 a $3000 \mathrm{~nm}$, ou 14300 a $3300 \mathrm{~cm}^{-1}$ ) constitui um campo ainda pouco explorado, em relação à região do IR médio, sendo esperado um avanço maior nesta área ${ }^{[17,18]}$. Existem na literatura estudos recentes sobre polímeros, onde são avaliadas as bandas na região $\mathrm{NIR}^{[19,20]}$. As bandas na região do infravermelho distante ${ }^{[21]}$ (FAR), especificamente na região de 700 a $200 \mathrm{~cm}^{-1}$, podem também ser úteis na caracterização da estrutura polimérica.

É conhecido que o uso de técnicas FTIR para caracterizar materiais não é algo novo, mas continua sendo uma ferramenta muito útil, aplicada recentemente à poliamidas ${ }^{[22]}$.

A avaliação de mudanças estruturais ocorridas em poliamida-6, reciclada e irradiada, por meio de várias técnicas, disponíveis em um único aparelho FTIR, no entanto, não é algo corriqueiro, sendo difícil encontrar em laboratórios de espectroscopia, e deve, portanto ser de interesse de profissionais da área de análise FTIR de polímeros.

Portanto, diante da possibilidade de utilização destes recursos, disponíveis em nosso laboratório (CTA), ou seja, um sistema espectrométrico com ampla faixa espectral (NIR, MIR, FAR), incluindo acessórios de análise de superfície (microscopia-FTIR) e PAS, o presente trabalho tem como objetivo dar continuidade à caracterização da poliamida-6, virgem, reciclada, e ir- radiada na presença de oxigênio, com feixe de eletron de 1,5 MeV, na dose de $500 \mathrm{kGy}$.

As amostras de polimida- 6 granuladas, virgem e reciclada, irradiadas a $500 \mathrm{kGy}$, foram analisadas por meio de diferentes técnicas de espectroscopia MIR, incluindo transmissão, reflexão (microscopia-FTIR), e PAS, associadas às bandas observadas na região NIR e FAR, para avaliar se existem mudanças significativas nas regiões FTIR de grupos amida, decorrentes da irradiação da poliamida.

\section{Experimental}

A poliamida-6 granulada virgem e reciclada, fornecida pela Radici Plastics, foi irradiada com feixes de elétrons provenientes de um acelerador linear eletrostático JOB 188 de 1,5MeV, na presença de oxigênio, com dose de $500 \mathrm{kGy}$, a uma taxa de $22,6 \mathrm{kGy} / \mathrm{s}$.

As análises FTIR foram conduzidas com o espectrômetro FTIR SPECTRUM 2000 Perkin-Elmer (resolução $4 \mathrm{~cm}^{-1}$; ganho 1; $4000 \mathrm{a} 400 \mathrm{~cm}^{-1}$ para análise MIR e até $250 \mathrm{~cm}^{-1}$ para a região FAR, 40 varreduras). Para análise MIR foram utilizados acessórios de microscopia-FTIR (modo reflexão, resolução 8, 128 varreduras) e PAS (resolução 4, velocidade $0,05 \mathrm{~cm}$, 4000 a $400 \mathrm{~cm}^{-1}, 32$ varreduras). Para a análise NIR foi utilizado o acessório de refletância difusa (DRIFT) ${ }^{[19,20]}$, (região 6000 a $4000 \mathrm{~cm}^{-1}, 47 \%$ de energia).

As amostras foram preparadas para as análises MIR e FAR por meio de diferentes técnicas de transmissão ${ }^{[8]}$ : filme vazado em ácido fórmico e pastilha de CsI (1,2:400 mg).

Para a análise por microscopia e PAS, as amostras foram preparadas como filmes vazados em ácido fórmico, tendo, respectivamente, como referência, "gold mirror" e negro de fumo.

Para análise DRIFT-NIR, as amostras foram preparadas sob a forma de filmes mais espessos que os da análise MIR, vazados em ácido fórmico.

\section{Resultados Discussão}

\section{Análise MIR}

As bandas no IR médio que caracterizam as poliamidas são devidas à ligação peptídica ${ }^{[8,23]} 3300 \mathrm{~cm}^{-1}$ ( $v$ do grupo $\mathrm{NH}), 1650 \mathrm{~cm}^{-1}$ ( $v$ do grupo $\mathrm{CO}-$ amida I), $1550 \mathrm{~cm}^{-1}$ ( $\delta$ do grupo $\mathrm{NH}+v$ do $\mathrm{CN}$ - amida II). Embora estas bandas falhem para diferenciar poliamidas 
de outros polímeros contendo grupos $\mathrm{NH}$, as bandas remanescentes exibem diferenças características, especialmente na região de 800 a $200 \mathrm{~cm}^{-1}$, permitindo a diferenciação de tipos de poliamidas.

Na poliamida-6, bandas em $1135 \mathrm{~cm}^{-1}$ e $934 \mathrm{~cm}^{-1}$ têm sido atribuídas às regiões amorfas e cristalinas ${ }^{[6,23]}$. A banda em $1135 \mathrm{~cm}^{-1}$ provavelmente ${ }^{[21]}$, deve ser atribuída à vibração complexa de grupo amida e cadeia metilênica e/ou combinação da deformação ( $\delta$ )NH e estiramento ( $v$ ) $\mathrm{O}=\mathrm{C}-\mathrm{N}$, e a de 934 $\mathrm{cm}^{-1}$, relativa à $\delta \mathrm{NH}$.

Embora existam evidências de tais bandas da poliamida-6, mudanças na estrutura, não podem ser concluídas decisivamente do seu espectro IR, nesta região. Entretanto, existe a citação na literatura ${ }^{[6]}$ de uma banda desenvolvida em $3450 \mathrm{~cm}^{-1}$, atribuída ao $v$ do grupo $\mathrm{COOH}$, quando a poliamida- 6 foi submetida à irradiação $\gamma$ na presença de ar. Embora esta absorção esteja realmente na faixa de grupo $\mathrm{COOH}$, considerando o histórico da amostra, um cuidado deve ser tomado: a técnica de pastilha de $\mathrm{KBr}$ utilizada para a preparação da amostra contribui para formação de grupo $\mathrm{OH}$ decorrente da umidade do $\mathrm{KBr}$, que absorve na mesma região. Deve ser considerado também que a amostra é higroscópica. Portanto, é recomendável a avaliação da região de $\mathrm{COOH}$ em torno de $1700 \mathrm{~cm}^{-1}$. Apesar da técnica de pastilha ser adequada à avaliação de cristalinidade ${ }^{[24]} \mathrm{em}$ polímeros, a técnica de transmissão preferida é a preparação de amostras sob a forma de filmes finos prensados a quente ou vazados de solução de solventes adequados ${ }^{[8]}$. Entretanto, a orientação e a cristalinidade dos polímeros podem ser alteradas.

Deste modo, com as devidas ressalvas, incluindo interações entre solventes e polímero, as amostras foram analisadas sob a forma de filmes. A Figura 1 inclui a comparação dos espectros IR destes filmes vazados em ácido fórmico de poliamida-6 virgem, reciclada e irradiada a $500 \mathrm{kGy}$. É interessante citar que além do alargamento da banda na região de $v$ do grupo $\mathrm{OH}\left(3450 \mathrm{~cm}^{-1}\right)$ ou $v \mathrm{NH}$ livre ${ }^{[8,9]}$, observa-se uma absorção em $1710 \mathrm{~cm}^{-1}$, mais evidentemente no espectro do filme vazado da amostra de poliamida- 6 reciclada irradiada, que sugere a presença do $v$ do grupo $\mathrm{COOH}$, provavelmente, formado pela irradiação, em presença de oxigênio, em concordância com Gupta e Pandey ${ }^{[6]}$, e não de ácido fórmico remanescente, desde que sua carboxila absorve em $1724 \mathrm{~cm}^{-1}$. É também observada outra banda em $1734 \mathrm{~cm}^{-1}$, re- gião de $v$ de grupos ácidos e éster no espectro da poliamida reciclada. Este conjunto de bandas sugere alteração na estrutura, com a formação de novos grupos, o que também está em acordo com dados relacionados a tratamentos fornecidos à poliamidas, que mostram a formação de grupos $\mathrm{C}=\mathrm{O}$ em amostras recicladas $^{[11]}$.

Como foi citado anteriormente, a reciclagem e irradiação favorecem à quebra de estruturas, o que sugere que a formação de grupos carboxila, possa estar associada com a possibilidade de haver cisão da ligação C-N do grupo amida, que é a mais fraca ligação da cadeia polimérica, em analogia às observações feitas por Gupta e Pandey ${ }^{[6]}$.

A banda em $3450 \mathrm{~cm}^{-1}$ pode também estar associada à presença do $v$ do grupo $\mathrm{NH}$ livre, devido ao deslocamento no equilíbrio de ponte de hidrogênio, característica de amidas ${ }^{[9]}$. Entretanto, como nesta região entre $3500-3300 \mathrm{~cm}^{-1}$ podem existir grupos $\mathrm{OH}$ e NH livres ou associados, havendo sobreposição, isto dificulta a atribuição da banda ${ }^{[25]}$.

Os diferentes graus de ponte de hidrogênio constituem um dos fatores citados para explicar alterações espectrométricas FTIR ocorridas em espectros de poliamidas tratadas, especialmente na região de grupos $\mathrm{C}=\mathrm{O}^{[11]}$.

Em adição, devido à técnica de transmissão, no caso desta análise filme vazado, ter a característica de mostrar o interior da amostra em maior proporção

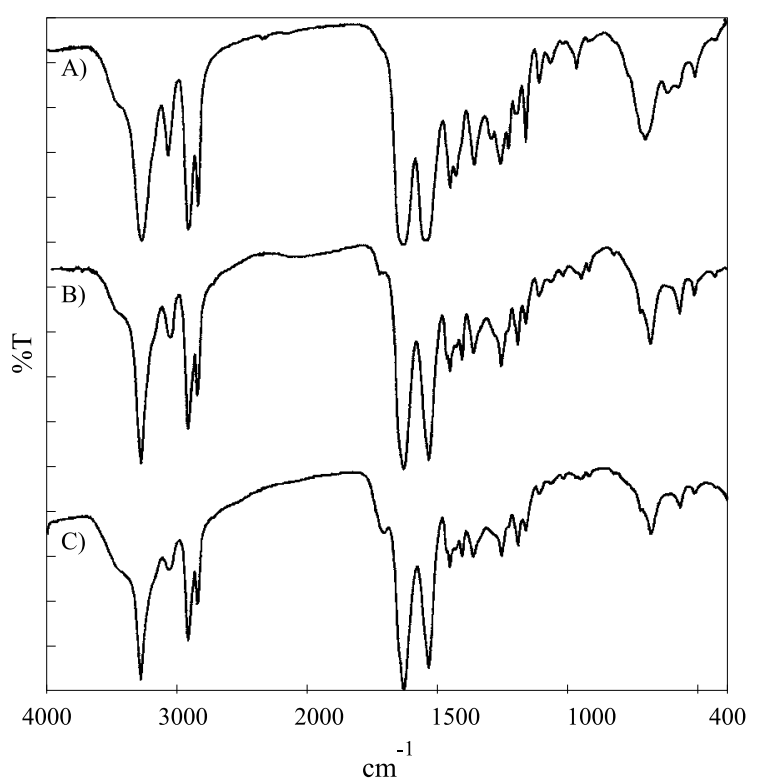

Figura 1. Espectros MIR (transmissão) de filmes vazados de amostras de poliamida, virgem, reciclada e irradiada a $500 \mathrm{kGy}$ 
que a espécie de superfície ${ }^{[8]}$, as alterações espectrométricas na região de grupos $\mathrm{C}=\mathrm{O}$, sugerem que o tratamento dado ao material atingiu o interior da amostra. Isto é consistente com os dados publicados por Cooper e colaboradores ${ }^{[12]}$ para a caracterização de superfície de filmes de poliamidas modificadas.

Outro estudo feito por Kelkar e Blalasubramanian ${ }^{[10]}$, sobre mudanças estruturais e elétricas em filme de poliamida-6, após tratamento com ion de gás inerte $\left(\mathrm{Ar}^{+}\right)$, mostrou alteração espectrométrica FTIR somente nas regiões de grupos $\mathrm{NH}\left(3500-2500 \mathrm{~cm}^{-1}\right)$ e $\mathrm{C}=\mathrm{O}$, sugerindo que algumas mudanças estruturais ocorreram envolvendo estes grupos, de modo similar ao que foi observado no estudo realizado em nosso laboratório para a poliamida -6 reciclada e irradiada.

\section{Análise PAS}

A técnica PAS utiliza deteç̧ão do sinal acústico de uma amostra gerada por meio de absorção de radiação modulada. A amostra é colocada numa pequena câmara, a qual um microfone é acoplado ${ }^{[26]}$. Radiação modulada é focalizada sobre a amostra, e certas freqüências que correspondem ao espectro de absorção do material são absorvidas. A radiação absorvida causa flutuações de temperatura da superfície.

Estas flutuações da temperatura de superfície da amostra induzem a mudanças periódicas de pressão do gás na célula fotoacústica. Uma onda sonora se desenvolve e é detectada por um microfone. Se uma freqüência particular não é absorvida, então a amostra não aquecerá e nenhuma onda sonora se desenvolverá. $\mathrm{Na}$ espectroscopia PAS, ondas sonoras são usadas para detectar freqüências de absorção de infravermelho. Amostras opacas são analisadas sem dificuldades.

Na Figura 2 são apresentados os espectros PAS das amostras de poliamida virgem, reciclada e irradiada a 500 KGy. Basicamente são observadas diferenças sutis, especialmente na região de grupos $\omega \mathrm{NH}$ $\left(600-750 \mathrm{~cm}^{-1}\right)$. É interessante citar que os espectros das amostras, reciclada e irradiada, apresentam região correspondente à impressão digital (1500 a 400 $\mathrm{cm}^{-1}$ ) similar, diferindo um pouco da amostra de poliamida virgem, o que está associado ao fato da amostra reciclada ser um material de partida da irradiada. Parece também existir um alargamento de bandas na região de $\mathrm{OH}$ e $\mathrm{COOH}$ (3450 e 1700-1740 $\left.\mathrm{cm}^{-1}\right)$ nestes espectros, de modo similar ao ocorrido nos espectros dos filmes vazados das amostras, obtidos por transmissão, porém de modo mais sutil.

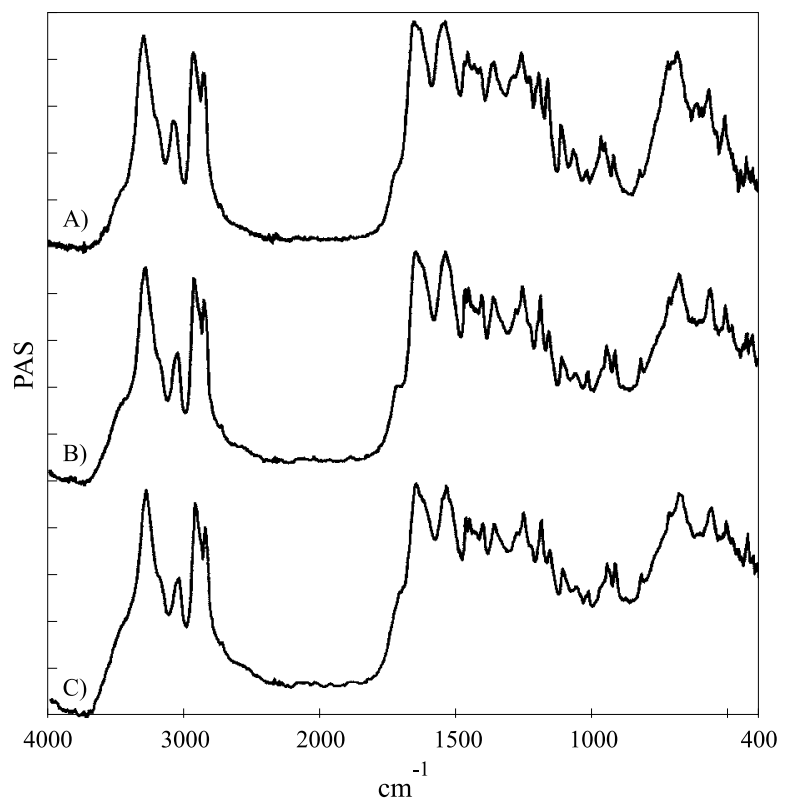

Figura 2. Espectros PAS-FTIR de filmes vazados de amostras de poliamida6, virgem, reciclada e não irradiada. A) Poliamica-6 virgem; B) Poliamida6 reciclada não irradiada; C) Poliamida-6 reciclada irradiada a 500kGy.

Cooper e colaboradores ${ }^{[12]}$, que utilizaram outra técnica de análise de superfície, reflexão total atenuada (ATR) para análise de filmes modificados de poliamidas, também observaram, somente alargamento dos modos vibracionais de poliamidas, o que atribuíram ao fato que ATR analisa, além da superfície, diversos micra no interior da amostra, sugerindo que o tratamento atingiu o interior do material.

No caso da técnica PAS, que foi utilizada neste trabalho, por ser mais adequada ao material opaco analisado, e que analisa a superfície mais próxima do material, é possível mostrar, devido às características da técnica FTIR, que houve alteração na camada superficial da amostra, causada pelo tratamento dado ao material. Entretanto, como citado anteriormente, a técnica de transmissão (filme vazado, figura 1), por ter a característica de mostrar o interior da amostra em maior proporção que a espécie de superfície, evidencia, desde que foram observadas alterações espectrométricas na região de grupos $\mathrm{C}=\mathrm{O}$, que o tratamento dado ao material atingiu também o interior da amostra.

\section{Análise por microscopia-FTIR}

A microscopia FTIR é essencialmente uma técnica para a análise de micro amostras ou áreas específicas de uma amostra ${ }^{[24]}$. O microscópio FTIR promove o exame visual e documentação da área que foi exposta à radiação IR. Este poder de combinação da imagem visual com o poder analítico da espectros- 


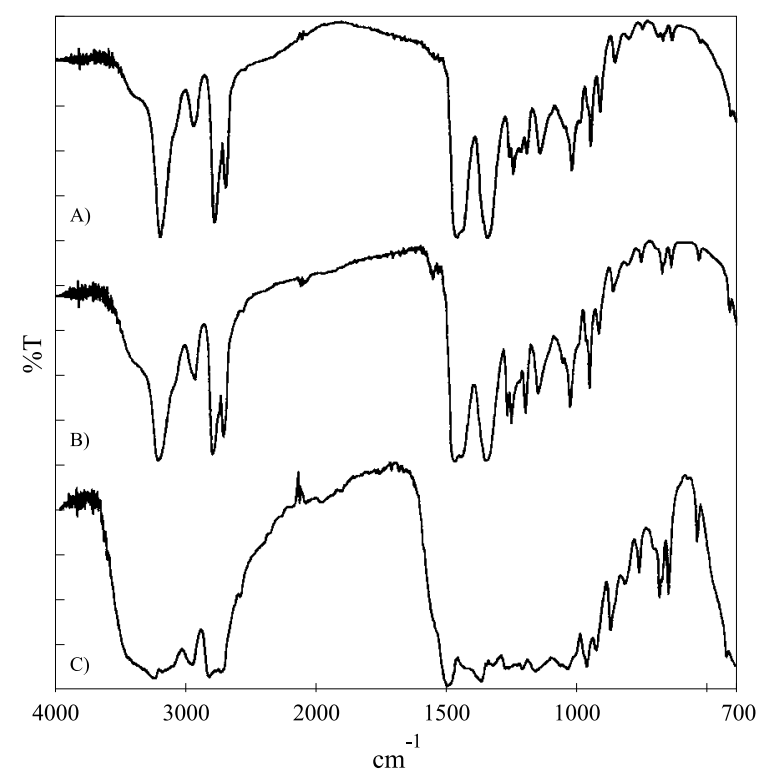

Figura 3. Espectros obtidos por Microscopia-FTIR de filmes vazados de amostras de poliamida-6 virgem, reciclada não irradiada e irradiada a 500kGy. A) Poliamida-6 virgem; B) Poliamida-6 reciclada não irradiada; C) Poliamida-6 reciclada irradiada a 500kGy.

copia FTIR confere o sucesso à esta técnica. Ambos os modos, transmissão e reflexão, são permitidos.

$\mathrm{Na}$ Figura 3 são mostradas as absorções obtidas por microscopia-FTIR dos filmes vazados das amostras de poliamida virgem, reciclada e irradiada. Parece haver também um alargamento de bandas nas regiões de grupos $\mathrm{OH}$ e $\mathrm{COOH}$, mais visível no espectro da amostra irradiada.

A região de impressão digital do espectro da amostra irradiada também apresenta absorções mais largas, similarmente ao observado por Cooper e colaboradores ${ }^{[12]}$ durante a análise ATR da superfície de filmes de poliamidas tratadas. Os pesquisadores atribuíram o alargamento de bandas na região de grupos $\mathrm{CN}$, incluídos portanto na região de impressão digital, ao decréscimo de cristalinidade.

\section{Análise FAR}

Absorções na região do infravermelho distante ${ }^{[21]}$ são devidas principalmente às vibrações de deformação do esqueleto, estiramento e deformação de ligações C-halogênio e C-Si.

As energias de transição são baixas, e as interações dentro e entre as moléculas podem ter uma forte influência na freqüência e intensidade de certas bandas de absorção. Isto implica numa sensibilidade de espectros FAR ao estado físico de uma substância e a conformação destas moléculas. Isto é vantajoso onde polímeros com a mesma estrutura química mas dife- rentes conformações ou taticidade ou cristalinidade devem ser analisados. As poliamidas podem cristalizar em diferentes formas polimórficas, portanto a análise na região FAR pode ser interessante. $\mathrm{O}$ aspecto desvantajoso da análise FAR em polímeros é que as interações ao acaso, dentro e entre moléculas polares, podem produzir alargamento de bandas. No infravermelho distante ${ }^{[21]}$ as bandas que provavelmente podem ser usadas para caracterizar o grupo amida em poliamidas são: $690 \mathrm{~cm}^{-1}$ (grupo $\mathrm{NH}$ ), 630-650, 625, $580 \mathrm{~cm}^{-1}$ (grupo C=O), $290-360 \mathrm{~cm}^{-1}$ (grupo CONC).

Na Figura 4 são comparados os espectros FTIR parciais $\left(800\right.$ a $\left.250 \mathrm{~cm}^{-1}\right)$ das amostras de poliamida virgem, reciclada e irradiada a $500 \mathrm{KGy}$ com espectro de poliamida 6 encontrado na literatura ${ }^{[21]}$. Embora não muito definidos, devido às intensidades características das bandas associado à redução da faixa espectral,
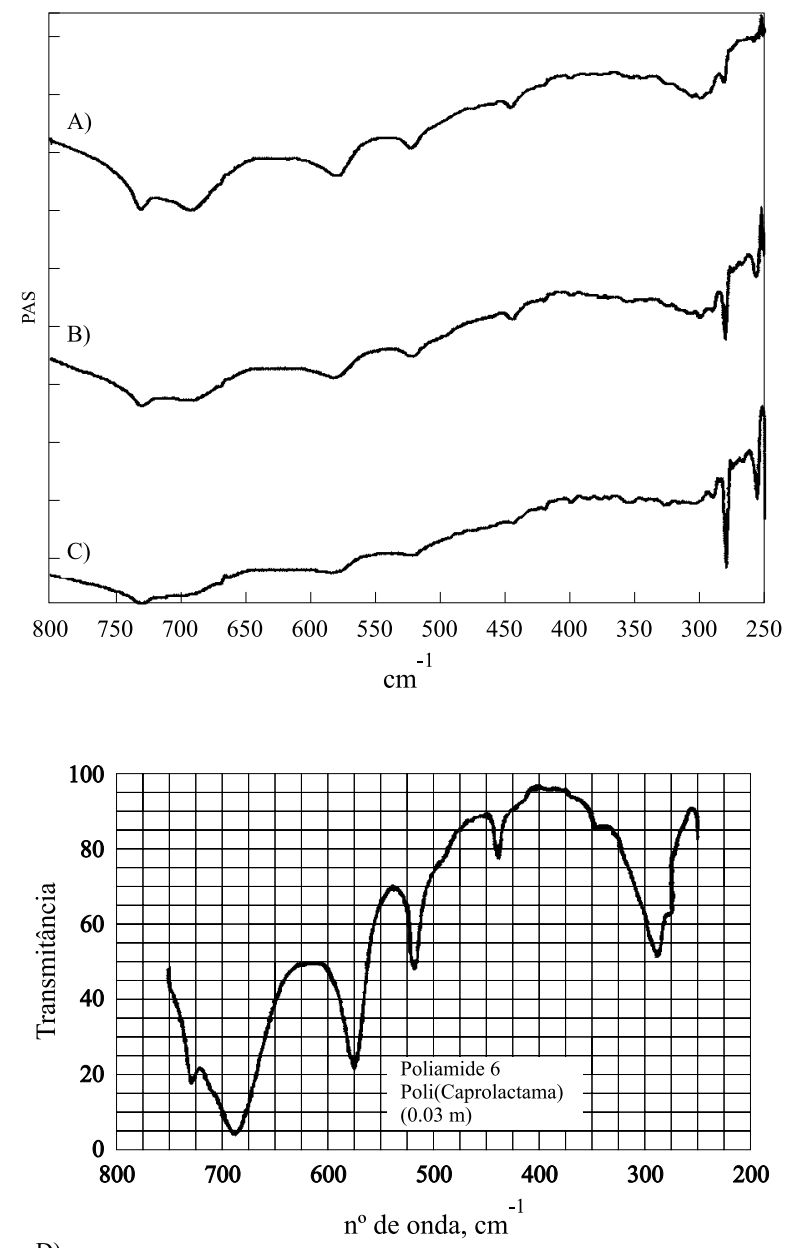

D)

Figura 4. Espectros parciais FAR $\left(800\right.$ a $\left.250 \mathrm{~cm}^{-1}\right)$ das amostras de poliamida-6 virgem e reciclada não irradiada e irradiada a $500 \mathrm{kGy}$. A) Poliamida-6 virgem; B) Poliamida-6 reciclada não irradiada; C) Poliamida-6 reciclada irradiada a 500kGy; D) Poliamida-6 - literatura. 
os espectros sugerem que há mudanças sutis que podem ser ou não devidas aos processos de reciclagem e irradiação. Pode-se observar que a amostra de poliamida virgem mantém as características do espectro de referência da literatura; entretanto existe maior alargamento de bandas nas regiões de grupos $\mathrm{NH}$ e $\mathrm{CONC}$ nos espectros das amostras de poliamida 6 , reciclada e irradiada a $500 \mathrm{KGy}$.

\section{Análise NIR}

O aparecimento de bandas que não são fundamentais na região NIR é explicado por meio da inarmonia do oscilador harmônico simples. Como as vibrações moleculares não são osciladores estritamente harmônicos, seus níveis de energia não estão separados de forma regular. Logo, transições entre níveis de energia diferentes são permitidas, originando os harmônicos ou sobretons, e as interações são possíveis, resultando em bandas de combinação. As freqüências dos sobretons são múltiplas das freqüências fundamentais e as de combinação correspondem à soma das freqüências de seus componentes ${ }^{[27]}$.

A maior energia da região NIR do que a do infravermelho médio excita sobretons e combinações de vibrações moleculares fundamentais ou não, tais como $\mathrm{C}-\mathrm{H}, \mathrm{O}-\mathrm{H}, \mathrm{N}-\mathrm{H}, \mathrm{C}=\mathrm{O}$, etc, em materiais, ao invés de excitar vibrações fundamentais ${ }^{[27]}$.

As absorções NIR são tipicamente 100 a 1000 vezes mais fracas que as bandas na região MIR; então, a radiação NIR deve atravessar 100 a 1000 vezes mais material para obter um espectro adequado. Por esta razão, medidas NIR são feitas, se possível, de soluções mais concentradas, ou de filmes mais espessos que os utilizados para a região $\mathrm{MIR}^{[27]}$.

Técnicas de análise de superfície podem também ser usadas em auxílio a de transmissão para uma avaliação mais completa do material ${ }^{[8,26]}$. Por exemplo a refletância difusa (DRIFT), onde a radiação difusamente espalhada é coletada por espelhos e direcionados ao detetor ${ }^{[26]}$. Em um experimento DRIFT, a radiação penetra no interior de uma amostra opaca e retorna após ter sido espalhada várias vezes. As reflexões de superfície e do interior da amostra são medidas ${ }^{[26]}$.

A técnica DRIFT-NIR já foi aplicada em trabalhos anteriores ${ }^{[19,20]}$ a amostras de poliamida 6 , recicladas. Resolveu-se então analisar as amostras de poliamida estudadas neste trabalho segundo metodologia similar. Na Figura 5 são apresentados os espectros das amostras de poliamida 6 , virgem e reciclada.

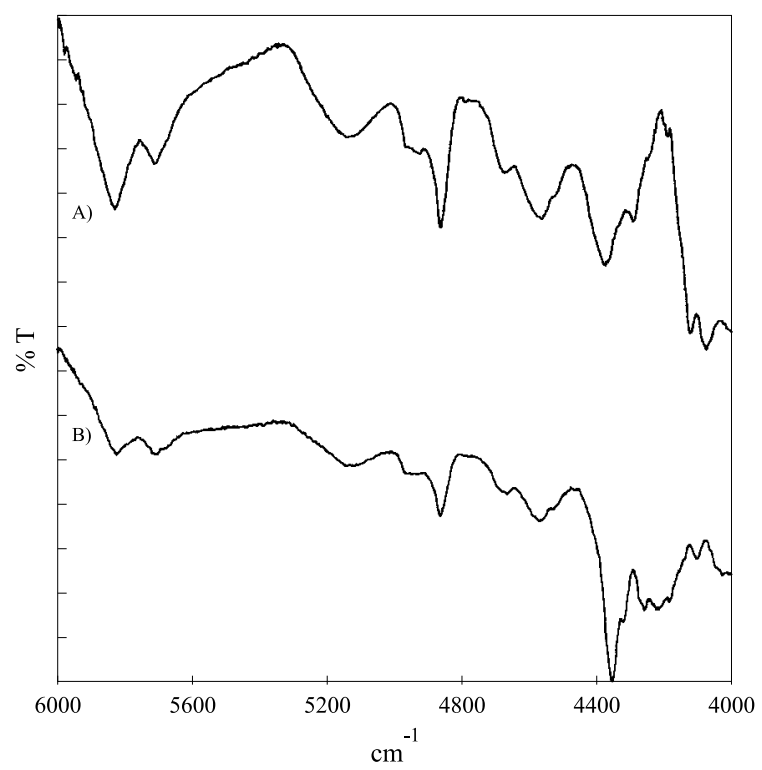

Figura 5. Espectros DRIFT-NIR de filmes vazados de amostras de poliamida-6 virgem e reciclada não irradiada. A) Poliamida-6 virgem; B) Poliamida-6 reciclada não irradiada.

A amostra irradiada formou um filme muito quebradiço, não permitindo análise adequada segundo esta técnica. Os espectros apresentam absorções que diferem em intensidade e posição na região de entre 4000 a $6000 \mathrm{~cm}^{-1}$, que inclui bandas de combinação e sobretons de absorções fundamentais de grupos $\mathrm{NH}$ em amidas ${ }^{[27]}$.

Como é conhecido, as técnicas FTIR apresentam diferenças quanto ao grau de profundidade de amostragem $^{[26]}$, sendo que entre as utilizadas neste trabalho, a microscopia-FTIR é a mais adequada à análise de superfície; as técnicas de transmissão e DRIFT, embora esta última seja também de análise de superfície, examinam também o interior da amostra.

Portanto, se foram observadas alterações espectrométricas com o uso destas técnicas de características diferentes, pode-se concluir que o tratamento atingiu tanto a superfície quanto o interior da poliamida-6.

Por outro lado, em apoio as diferenças sutis observadas nos espectros FTIR, foi encontrado na literatura $^{[28]}$ que para polímeros alifáticos, tais como poliamida-6, raramente existiu alguma diferença relacionada à taxa de radiação de próton e elétron nas propriedades mecânicas destes materiais.

\section{Conclusão}

A avaliação de amostras de poliamida 6, virgem, reciclada e irradiada a $500 \mathrm{KGy}$, por meio de técni- 
cas MIR de transmissão, reflexão (microscopiaFTIR), detecção fotoacústica, DRIFT-NIR e FAR, dentro dos seus limites de detecção, sugeriu que exista a formação de grupos $\mathrm{OH}$ e $\mathrm{COOH}$ e/ou $\mathrm{C}=\mathrm{O}$ nas amostras, reciclada e irradiada a $500 \mathrm{KGy}$, de forma mais evidente na amostra irradiada. Existem ainda indícios de mudanças de estrutura, relacionadas com grupos $\mathrm{NH}$ e -CN-C=O. Em concordância com estudos realizados para poliamidas, que citam que fatores ${ }^{[11]}$ como pontes de hidrogênio, decréscimo de cristanilidade podem estar ocorrendo.

A técnica FTIR-MIR de transmissão (filme vazado) e microscopia-FTIR mostraram de forma mais evidente as mudanças estruturais, embora a técnica PAS, por não ter característica de reflexão, e também mostrar alteracões espectrométricas, mesmo de forma mais sutil, pode ser considerada também como uma metodologia atrativa para a análise de corpos de prova opacos de poliamidas.

Finalmente, se foram observadas alterações espectrométricas com o uso destas técnicas de características diferentes, pode-se concluir que o tratamento atingiu tanto a superfície quanto o interior da poliamida-6.

\section{Referências Bibliográficas}

1. Clegg, D.W.; Collyer A. A.-"Irradiation Effects on Polymers”, Elsevier Applied Science, London, (1991).

2. Encyclopedia of Polymer Science Engeneering, v13, (1988).

3. Rosa, M. C. F. - "Preparação de compostos plásticoscelulósicos utilizando radiação", Dissertação e Tese IEA-DT-069 - Instituto de Energia Atômica, São Paulo, (1978).

4. Wiebeck H.; Bernardo, A.; Martini A. M.; Asciutti,S. A.; Oliveira,M.G.; Yai, C. H.- "Modificação das Propriedades Mecânicas da Poliamida-6 em reciclagens sucessivas". In: VI Simpósio Latino Americano de Polímeros, IV Congresso Iberoamericano de Polímeros, IV Simpósio Chileno de Química y Fisicoquímica de Polímeros, October 25-28, 1998, Viña del Mar-Chile. Libro de Resúmenes...Santiago: Sociedad Chilena de Química, p. 200, (1998).

5. Singh, L.P.; Chaudhuri, N.K.- "Effect of gamma irradiation on nylon 6 films", Proceedings of Symposium on Industrial Polymers and Radiation, Sardar Patel University, Vallabh Vidyanagar, Gujarat, February 12-14, (1979).
6. Gupta, M.C.; Pandey,R.R. - "g- Irradiation of Nylon 6", Journal of Polymer Science, v 26, p. 491-502, (1988).

7. Evora, M. C.; Gonçalez, O . L.; Wiebeck, H.; Machado, L. D. B.; Andrade E Silva, L. G.- "Análise dos efeitos da radiação ionizante sobre a poliamida-6 reciclada por termogravimetria e calorimetria exploratória diferencial". In: $2^{\circ}$ Congresso Brasileiro de Análise Térmica e Calorimetria, $1^{\circ}$ Congresso Panamericano de Análise Térmica e Calorimetria, April 9-13, 2000, Poços de Caldas-Minas Gerais-Brazil. Livro de resumos p. 186, ABRATEC, São Paulo, (2000).

8. Smith, A. L. - "Applied Infrared Spectra”, John Wiley \& Sons, New York, (1979).

9. Jawhari, T.; Quintanilla, L.; Pastor, J.M.- "A Comparison of Specular Reflection and PA-FTIR Techniques in the Analysis of Annealed Injection-Molded Poliamide 6,6", Journal of Applied Polymer Science, v 51, p 463-471 (1994).

10. Kelkar, D.S.; Balasubramanian, V.- "Electrical and Structural Properties of $\mathrm{Ar}^{+}$Implanted Nylon-6 Films", Polymer International v42, p 393-396 (1997).

11. Eriksson, P. A; Boydell, P.; Eriksson, K.; Manson, J. A.E.; Albertsson, A. C. - "Effect of Thermal-Oxidative Aging on Mechanical, Chemical, and Thermal Properties of Recycled Polyamide 66", Journal of Applied Polymer Science, v 65, p.1619-1630, (1997).

12.Cooper, J.B.; Julian, B,; Morrison, H.; Song, P.; Albin, $\mathrm{S}$,; Zheng, J,- "Surface characterization of pulsed UV-laser modified polyamide films".

13. Dutra, R.C.L, Takahashi, M.F.K., Diniz, M.F. "Importância da Preparação de Amostras em Espectroscopia no Infravermelho com Transformada de Fourier (FTIR) na Investigação de Constituintes em Materiais Compostos", Polímeros: Ciência e Tecnologia, ano V, n 1, jan/mar, p.41-46, (1995).

14. Dutra, R.C.L.; Soares, B.G.; Campos, E.A., Melo, J.D.G.; Silva,J.L.G. - "Composite Materials Constituted by a Modified Polypropylene Fiber and Epoxy Resin", Journal of Applied Polymer Science, $v$ 73, p.69-73, (1999).

15. Ishida, H. -"Quantitative surface FTIR spectroscopy analysis of polymers, Rubber Chem. Technol., p. 497-554, (1987).

16. Patel, S. H.; Todd, D. B.; Xanthos, M. - "Antec, Recent developments in on-line analytical techniques applicable to the polymer industry”, p. 2214-2219, (1994). 
17. Varley, R. J; Heath, G. R.; Hawthorne, D. G.; Hodghin, J. H.; Simon, G. P.- " Toughening of a trifunctional epoxy system: 1. near infrared-red spectroscopy study of homopolymer cure", Polymer 36, 7, p. 1347-1355, (1995).

18. Mijovié, J.; Andjelié, S.; Yee, C .F. W.; Belluci, F.; Nicolais, L. - "A study of reaction kinetics by nearinfrared spectroscopy.2.Comparison with dielectric spectroscopy of model and multifunctional epoxy/ amine systems", Macromolecules, v 28, p. 2797 2806, (1995).

19. Araújo, S.C.; Kawano,Y.; Wiebeck; H.; Batista, M.S. "Identificação de poliamida 6 reciclada utilizando espectroscopia no infravermelho próximo", 50 Congresso Brasileiro de Polímeros, Anais CBPol, 135, p. 565-566, São Paulo, Brasil, (1999).

20. Araújo, S.C.; Kawano,Y.; Wiebeck; H.- "Identificação de poliamida 6 reciclada utilizando espectroscopia no infravermelho próximo", VI Simpósio LatinoAmericano de Polímeros - IV Congresso Iberoamericano de Polímeros- IV Simpósio Chileno de Química e Físico Química - Chile, p. 1-46, (1998).

21. Hummel, D.O - "Infrared Spectra of Polymers in medium and long wavelength regions", Interscience Publishers, New York, p. 101, (1967).
22. Lin, J.; Cammarata,V.; Worley, S.D. - "Infrared characterization of biocidal nylon", Polymer v42, 79037906, (2001).

23. Urbanski, J. - "Handbook of Analysis of Synthetic Polymers and Plastics", John Wiley \& Sons, New York, (1977).

24. Graf, R. T. ; Koenig, J. L.; Ishida, H. - "Introduction to optics and infrared spectroscopic techniques", Polym. Sci. Technol., v 36, p. 1-31, (1987).

25. Serpe, G.; Chaupart, N. - "Relaxation- Structure Relationship in Bulk and Plasticized Polyamide 11", Journal of Polymer Science PartB: Polymer Physics, v.34., p 2351-2365, (1996).

26. Pandey, G.C.; Kulshreshtha, A .K. - "Fourier transform infrared spectroscopy as a quality control tool", Process Control and Quality, v 4, p. 109-123, (1993).

27. Kathryn, A.; Bunding,L. - "Comparison of Mid-IR with NIR in Polymer Analyis", Applied Spectroscopy Reviews, v.28, 3, p. 231-284, (1993).

28. Sasuga, T.; Kawanishi, S.; Nishii, M.; Seguchi, T.; Kohno, I. - "Effects of ion irradiation on the mechanical properties of several polymers" - Radiation Physics and Chemistry 1991, v. 37, 1, p. 135-140.

Recebido: $15 / 12 / 01$ Aprovado: 10/04/02 